\title{
ON THE THEORY OF IMPROPER DEFINITE INTEGRALS*
}

\author{
BI \\ ELIAKIM HASTINGS MOORE
}

$\S 1$.

INTRODUCTION. THE FOUR CURRENT TYPES OF IMPROPER INTEGRALS.

$1^{\circ}$. In this paper I wish to define a system of types of improper simple definite integrals, a system embracing in particular the four current types; of the theory of the general type I give at present merely the elements, the methods employed, however, being characteristic.

The four current types are compared in $\S 12^{\circ}-13^{\circ}$. By way of generalization of their diversities the new types arise $\left(11^{\circ}-17^{\circ}\right)$. As the desirable basis for the new types I propose $\left(16^{\circ}\right)$ an extension of the notion of the proper simple definite integral ; this involves likewise an extension of the notions of the four current types.

In $\S 2$ I state in convenient notations a body of elementary properties of the general type of integrals. These properties with two definitional processes of induction developed in $\S \S 3,4$ serve as the basis for the definition in $\S 5$ of the system of types of improper integrals related to the (extended) type of proper integrals defined in $16^{\circ}$.

$2^{\circ}$. If the definite integral

$$
\int_{a}^{b} F(x) d x
$$

is a proper integral, the interval $a b$ of integration is finite and the integrand function $F(x)$ exists (everywhere defined but not necessarily in a single valued way) on $a b$ as a limited function.

$3^{\circ}$. IMPROPER INTEgRals OVER Finite INTERVALS.-We call to mind the four current types of improper integrals (1) over finite intervals $a b$. The following presuppositions are common to the four types. On $a b$ the function $F(x)$ has a singular point-set $Z$. In every neighborhood of a point $\zeta$ of $Z$ the function $F(x)$ is unlimited. On every interval of $a b$ containing no point $\zeta$ the function $F(x)$ is properly integrable.

\footnotetext{
* Presented to the Society at the Ithaca meeting August 19, 1901. Received for publication Ootober 2, 1901.
} 
$4^{\circ}$. Trpe I.-In the first type the set $Z$ is reducible, that is, for some (lowest) ordinal * $a$ of CANTOR's first or second class of ordinals, the derived set $Z^{(a)} \equiv 0$; hence the set $Z$ has the content zero, and it is finite or numerably infinite; and conversely, any set finite or numerably infinite is reducible. CAUCHY and RIEmann considered the case of finite sets $Z(a=1)$, and DU Bors-REYMond (1875: Crelle's Journal, vol. 79, pp. 36, 45), and Dini (1878: DinILüroth, pp. 404-445) the case of sets $Z$ of the first kind $\dagger(a=\nu=1,2,3, \cdots)$, and quite recently Schoenfles (1900: Bericht, pp. 185-186, Jahresbericht der Deutschen Mathematiker Vereinigung, vol. 8, no. 2) the general case $(a=a)$.

$5^{\circ}$. TyPe II.-In the second type the set $\mathrm{Z}$ is any set of content zero. For a systematic exposition of the theory of these the (general) HARNack integrals of 1883-1884 I refer to my paper in the last number of this journal (pp. 296-330); in the introduction are indicated the relations with the equivalent JORDAN theory of 1894.

The theories of these two types have this fundamental difference: the integral of the second type is defined by a definition covering all cases at once, while all cases of the first type are covered in that the integral for the case $a=1$ is defined directly and then two definitional processes are given which serve to define, in the first place, the integral for the case $a=a_{0}+1$ in terms of integrals for the case $a=a_{0}$ and, in the "second place, the integral for the case $a=a_{\omega}$ in terms of a numerably infinite set of integrals for the cases $a=a_{\nu}(\nu=1,2,3, \ldots)$, where the ordinal $a_{\omega}$ is next higher than the set $\left\{a_{\nu}\right\}$. Thus the definition of a particular integral of the second type involves a single limiting process, and that of one of the first type involves a finite or a numerably infinite system of limiting processes.

$6^{\circ}$. In the comparison of two types of integrals, presupposing that in each theory every integral (1) when existent has a definite finite value, we say for brevity that the two types are accordant or in accord in case every integral existent in each theory has in the two theories equal values; and of two accordant types we say that the first contains or includes the second if every integral existent in the second theory exists also in the first. Two types are equivalent if each includes the other.

$7^{\circ}$. Now to compare the first two theories, we see that the second theory has wider application than the first theory, since the second theory covers the cases of irreducible sets $Z$. As to integrals with reducible sets $Z$, the two types are

* That is ordinal number (Ordinalzahl).

$\dagger$ Gattung. 
well known to be equivalent for the case $a=1$. For the cases* $a>1$, however, while it is readily seen that the first type contains the second, the two types are not equivalent ; for example $(a=2)$, the integral (31) p. 330 of my paper cited above exists or not according as it is of the first or second type.

$8^{\circ}$. TYPE III.-The third type is that of HöLder (Mathematische Annalen, vol. 24 (1881), p. $90 \mathrm{ff}$.); the set $\mathrm{Z}$ is reducible. Hölder defines primarily an indefinite integral

$$
\int F(x) d x !
$$

on the $x$-interval $a b$, viz., as any function $\phi(x)$ continuous on $a b$ and such that for any two points $x^{\prime} x^{\prime \prime}$ of $a b$, whose interval $x^{\prime} x^{\prime \prime}$ contains no point $\zeta$ one has the relation

$$
\phi\left(x^{\prime \prime}\right)-\phi\left(x^{\prime}\right)=\int_{x^{\prime}}^{x^{\prime \prime}} F(x) d x .
$$

This definition would apply to any set $Z$. The restriction to reducible sets $Z$ is made in order that two such indefinite integrals on $a b$ may differ on $a b$ by a constant, and thus that the definite integral:

$$
\int_{a^{\prime}}^{b^{\prime}} F(x) d x=\phi\left(b^{\prime}\right)-\phi\left(a^{\prime}\right),
$$

where $a^{\prime} b^{\prime}$ are any two points of $a b$, may be independent of the indefinite integral $\phi(x)$ used in its definition.

The first and the third types of definite integrals are easily seen to be equivalent. Indeed it is possible that HöLDER had in mind the final extension, later made by Schoenflies, of the integrals of the first type to the case of any or$\operatorname{dinal} a$.

$9^{\circ}$. Type IV.-The fourth type is that of De la VAllÉe-Poussin (1892: Liouville's Journal, ser. 4, vol. 8, pp. 421-467). For the general set $Z$ of content $\dagger$ zero a primary definition is given by a single limiting process which is of such a nature that the integral (1) if existent is absolutely existent. Then, on

* I have found only the two following notes of comparison of the two types for these cases:

SToL" (Wiener Berichte, vol. 107.a (1898), pp. 207-224: ef. \$2) states that for the case $a=\nu>1$ the second type is not evidently (" nicht ohne Weiteres") to be identified with the first.

Schornflies (loc. cit., p. 188, 11. 21-24) discriminates in general the cases of sets $\mathrm{Z}$ reducible and irreducible by introducing the terms: integrals of the first and second kinds (Arten), and thus distinctly implies that for reducible sets $\mathrm{Z}$ the two types are equivalent.-The proper disorimination is by the type.

† Schoenfles (loc. cit., pp. 178, 187, §5) points out and emphasizes the fact that this condition if not inserted in the definition may be deduced as a conclusion, in any case in which the (modified) definition yields an integral. 
the basis of the proper and of these absolutely existent improper integrals, for the general reducible set $Z_{0}$ of all points $\zeta$ of $Z$ in whose neighborhood the function $F(x)$ is not absolutely integrable the non-absolutely existent integrals are defined ${ }^{*}$ as in the first theory.

It is to be noted that Schoenflies (loc. cit., pp. 188, 189) gives vague indications that the case of irreducible sets $Z_{0}$ may be included by suitable phrasing of the secondary definition, and indeed that the primary definition may be so modified as to include the non-absolutely existent integrals.

The second and the fourth types are equivalent in so far as absolutely existent integrals are concerned, $\dagger$ and, likewise, as to non-absolutely existent integrals, in the case $a=1$ for the reducible set $Z_{0}$, while in the cases $a>1$ the fourth type contains but is not equivalent to the second type; the example (for $a=2)$ cited above $\left(7^{\circ}\right)$ serves here also.

$10^{\circ}$. To summarize: The Harnack (second) type of integral (1) has the widest range of application and it involves a single limiting process. The other types involve in general a system of limiting processes. Every type contains the corresponding subtype of the HaRNack type. Every subtype involving only one limiting process is equivalent to the corresponding HaRnaCK subtype.

In every theory the integral symbol (1) has a unique definition.

$11^{\circ}$. For a given singular set $\mathrm{Z}$ the system of types of improper integrals (1) to be defined in this paper arises from the four current types by way of generalization of their diversities. The reader will doubtless have foreseen the character of the general type to be proposed. It bears to the HARNACK type a relation which generalizes (not uniquely) the relation borne by the first type $(a=a)$ to the CAUCHY subtype $(a=1)$. The integral symbol (1) for any type of the system receives a unique definition depending on the type, or, otherwise expressed, on the way in which the set $\mathrm{Z}$ is utilized in the limiting process or processes involved in the definition.

At present in connection with the definition of the system of types I consider briefly the theory of the general type of the system but not the relations amongst the various types. I conjecture however that this relation is (as amongst the current types) simply that every type is in accord with every other type. If this relation is indeed valid, we may obviously unite or fuse all the types of the system into a new single type in which the symbol (1) has again a unique definition. $\dagger$

* The definition given by de LA VAllée-Poussin (loc. cit., p. 453, §55) is restricted to the case of reducible sets $\mathrm{Z}_{0}$ of the first kind.

$\dagger$ Cf. STOLZ (loc. cit., introduction).

$\ddagger$ It in any particular type of the system a symbol (1) has a value, it shall have that value in the new type. If in every type of the system the symbol (1) has no value, it shall have no value in the new type. 
$12^{\circ}$. Since in the theory of a particular type of integrals the definite integral function

$$
J(x)=\int_{a}^{x} F(x) d x
$$

$(x$ on $a b)$

of a function $F(x)$, properly or improperly integrable from $a$ to $b$, is a continuous function of $x$ on $a b$, and quâ $\phi(x)$ satisfies the relation (3), we have in that theory, even for irreducible sets $Z$, means of selecting from amongst all HöLDER's functions $\phi(x)$ a system every two of which differ on $a b$ by a constant, viz., the system

$$
\phi(x)=J(x)+C,
$$

where $C$ is an arbitrary constant. For this theory these functions $\phi(x)$ may then properly receive the indefinite integral notation:

$$
\phi(x)=\int F(x) d x .
$$

In case we may fuse all the types of the system into a new type, as suggested, in $11^{\circ}$, we obtain in the new theory a unique system of indefinite integrals (7).

$13^{\circ}$. IMPROPER INTEGRALS OVER INFINITE INTERVALS. - In each of the four current types the improper integral (1) over an infinite interval $a b$ is defined either explicitly or presumptively as the limit of the proper or improper integral over the finite subinterval $a^{\prime} b^{\prime}$ of $a b$ as $a^{\prime} b^{\prime}$ converges to $a b .^{*}$ Thus for each type the theory of the improper integrals over infinite intervals depends upon that of the improper integrals over finite intervals.

It is, however, possible to define the former integrals as transforms of the latter integrals. The transformation

$$
x=\psi(x)=-(\vec{x} \pm 1)^{-1} \pm 1 \quad\left(x \text { on the interval }{ }_{0}^{-\infty} \cdots \infty\right)
$$

throws the complete $\dagger x$-line $(-\infty \leqq x \leqq \infty)$ in a one-to-one way on the $\bar{x}$-interval $-1 \leqq x \leqq+1$; as $x$ traverses the $x$-line in the positive sense $x$ traverses the $x$-interval in the same sense; $\psi(\bar{x})$ is a continuous function of $\bar{x}$ with a continuous positive derivative $\psi^{\prime}(\bar{x})=(\bar{x} \pm 1)^{-2}$ on the $x$-interval excluding its extremities $\mp 1$. Then, setting

* We define in like manner the content $\gamma(\Xi)$ of a point-set $\Xi$ lying on an infinite interval $a b$ as the limit of the content $\gamma\left(\Xi^{\prime}\right)$ of the subset $\Xi^{\prime}$ of $\Xi$ lying on the finite subinterval $a^{\prime} b^{\prime}$ of $a b$ as $a^{\prime} b^{\prime}$ converges to $a b$.

$\dagger$ It is here permissible and convenient to admit the definite infinities $\infty,-\infty$ as arguments and functional values. For a function $f(x)$ the two numbers

$$
f(\infty), f(\infty-0) \equiv \underset{x=\infty}{\mathbf{L}} f(x)
$$

are definitionally distinct, and so are $f(-\infty), f(-\infty+0)$. The function $f(x)$ is continuous at $\infty$ if $f(\infty), f(\infty-0)$ exist and are equal. 


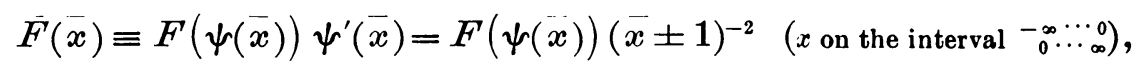

and denoting by $a, \bar{a}$ and $b, \bar{b}$ two pairs of corresponding values of $x, \bar{x}$ respectively, we have the relation of transformation:

$$
\int_{a}^{b} F(x) d x=\int_{\bar{a}}^{\bar{b}} F(\bar{x}) d x .
$$

This relation is valid for finite intervals $a b$.-We may use the relation to define the integrals over infinite intervals, viz., as transforms of those over finite intervals:

Similarly, for any type of integrals, we may define the integrals over infinite intervals either as limits of integrals of the same type or as transforms of integrals of a transformed type, these integrals being in each case over finite intervals.

It seems to be desirable to adopt the former and usual definition as the definitive definition for use in the applications of the theory of any type of integrals, but in the development of the theory to adopt the latter new definition. For it is evident that an integral under the new definition is one under the old definition, while an integral under the old definition, if not one under the new definition, is one under the new definition for a closely related type of integrals ; and so the theory is richer by the adoption of the old definition while its development (if made for the general type of integrals) is somewhat simpler by the adoption of the new definition.

For the first, third, and fourth types the two definitions are equivalent. In the second type, however, it is possible to have, for example, a HARNACK integral $\int_{1}^{\infty} F^{\prime}(x) d x$ (according to the old definition) whose transformed $\mathrm{H}_{\mathrm{AR}}$ NACK integral $\int_{\frac{1}{2}}^{1} \bar{F}(\bar{x}) d \bar{x}$ does not exist: the example of $\S 39^{\circ}$ of my paper already cited is to the point, - on the $x$-interval $\frac{1}{2} 1=a b$ the present function $\bar{F}(x)$ being the function $F^{\prime}(x)+G(x)$ of the reference.

$14^{\circ}$. For the sake of clearness of thought and also of greater generality we replace the point-set $\mathrm{Z}$ of singularities $\zeta$ of the integrand function $F(x)$ by any point-set $\Xi$ of content zero.* The general type of integral is then in a certain sense a function or an aspect of $\Xi$. The integral (1) depends upon the type, the interval $a b$, and the integrand function $F^{\prime}(x)$. If the symbol (1) has value, the function $F(x)$ has certain relations to $\Xi$, e. g., on every interval of $a b$ containing no point $\xi$ of $\Xi$ the function $F(x)$ is properly integrable; and further, the limiting relations implied in the type hold.

$15^{\circ}$. For the purpose of the later theory the content $\gamma(\Xi)$ of $\Xi$ needs to be zero. But the definitions and theory of the present paper are effective also for

\footnotetext{
* But cf. $15^{\circ}$.
} 
sets $\Xi$ of arbitrary content $\gamma(\Xi)$; since this fact has interest, the more general form of presentation is adopted.

$16^{\circ}$. The more general Proper Definite integrals.-I extend the notion of the proper definite integral (1) as follows:

The interval $a b$ is a finite interval. The integrand function $F(x)$ exists on $a b$, or, at least, on $a b$ apart from a point-set $\Omega$ of content zero, as a function everywhere defined* and limited ; $\dagger$ and a function $G(x)$, arising from $F(x)$ by modification of its determination on $\Omega$ so that $\dot{G}(x)$ is on $a b$ everywhere defined and limited, is properly integrable from $a$ to $b$ in the usual sense. Then we set in general

$$
\int_{a}^{b} F(x) d x=\int_{a}^{b} G(x) d x
$$

In case on the set $\Omega F(x)$ itself is everywhere defined and limited, the integral (1) exists as a proper integral in the usual sense, and the equality (11) is well known.

In other cases the equality (11) serves to define the integral (1) as a definite finite number, a proper definite integral in the new sense.

By virtue of this definition of the type of proper integrals every existing definite integral (1) of this type or of any derivative type defined in this paper (\$5) retains its type and value under any modification of the determination of the integrand function on a point-set of content zero. This statement is embodied in theorem II of $\S 2$; and it is to be compared with the remark of $\S 5$ $7^{\circ}$ (p. 329) of my paper cited above $\left(5^{\circ}\right)$.

\section{$\S 2$.}

Notations and elementary PROPERTIES OF THE GENeral TYPE OF INTEGRALS.

$1^{\circ}$. With respect to any linear point-set $\Xi\left(\S 11^{\circ}\right)$ we are to consider a system of types of improper definite integrals $(\S 1(1))$, and especially the general (any particular) type of the system. For this type we use the notation I (" integral"), instead of the integral sign $\int$ with an affix indicating the type in question. Thus, the symbol:

$$
\mathbf{I}_{a}^{b} F(x) d x,
$$

where $a$ and $b$ are definite points (finite or infinite) and where $F(x)$ is a definite

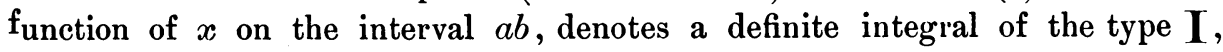

* Not necessarily in a single valued way.

† On the set $\Omega F(x)$ need not be defined, and, so far as it is defined, it need not be limited. 
which is either existent and of definite finite value or non-existent. We speak of the I-integral (1) and of functions $F(x)$ I-integrable from $a$ to $b$.

The closed set $\Xi+\Xi^{\prime}$ is supposed to contain the two infinite points $( \pm \infty)$. If $\Xi=\{+\infty,-\infty\}$ the (only) type $\mathbf{I}$ of the set $\Xi$ is the type of the proper definite integrals of $\S 116^{\circ}$ over finite intervals and

The integrals of type $\mathbf{I}$ over subintervals of a fixed interval $a b$ constitute a type $\mathbf{I}_{a b}$. With respect to the type $\mathbf{I}_{a b}$ all sets $\Xi$ having the same subset $\Xi_{a b}$ lying on $a b$ are equivalent.

$2^{\circ}$. The following theorems (I-VIII) of the theory of the proper definite integrals defined in $\S 116^{\circ}$ hold also in the theory of the integrals of the type $\mathbf{I}$.

$\mathbf{I}^{*}$. The function 0 is $\mathbf{I}$-integrable from $a$ to $b$, and $\mathbf{I}_{a}^{b} 0 d x=0$.

II. A change in the determination of the integrand function $F(x)$ in the points of a point-set of content $\dagger$ zero makes no change in the determination of the $\mathbf{I}$-integral $\mathbf{I}_{a}^{b} F(x) d x$.

FIXED HYPOTHESIS.-The function $F(x)$ in III-VIII (IV' apart) is supposed to be I-integrable from a to $b$.

III. The function $F(x)$ is likewise I-integrable from $x_{1}$ to $x_{2}$, where $x_{1} x_{2}$ are any two points of $a b$.

IV. For any three points $x_{1} x_{2} x_{3}$ of the interval $a b$

and accordingly

$$
\mathbf{I}_{x_{1}}^{x_{2}} F(x) d x+\mathbf{I}_{x_{2}}^{x_{3}} F(x) d x=\mathbf{I}_{x_{1}}^{x_{3}} F(x) d x ;
$$

$$
\mathbf{I}_{x_{1}}^{x_{1}} F(x) d x=0, \quad \mathbf{I}_{x_{2}}^{x_{1}} F(x) d x=-\mathbf{I}_{x_{1}}^{x_{2}} F(x) d x .
$$

IV'. If $F^{\prime}(x)$ is $\mathbf{I}$-integrable from $x_{1}$ to $x_{2}$ and from $x_{2}$ to $x_{3}$, then it is $\mathbf{I}$-integrable from $x_{1}$ to $x_{3}$, and the equality of IV holds.

V. For every positive number $\epsilon$ there exists a positive number $\delta_{\epsilon}$ such that $\left|\mathbf{I}_{x_{1}}^{x_{2}} F(x) d x\right|<\epsilon$ for every two points $x_{1} x_{2}$ of ab for which $\left|x_{1}-x_{2}\right|<\delta_{\epsilon}$.

In the light of theorem III, theorem $\mathrm{V}$ is the affirmation of the uniform continuity on $a b$ of the definite integral function

$$
J(X)=\mathbf{I}_{a}^{X} F(x) d x \quad(X \text { on } a b),
$$

an affirmation known to include and to follow $\ddagger$ from the statement:

$\mathrm{V}^{\prime}$. The definite integral function $J(X)(2)$ is a continuous function of the variable upper limit $X$ on the $X$-interval $a b$.

VI. The function $c F(x)$, where $c$ is any constant, is $\mathbf{I}$-integrable from $a$ to $b$, and

$$
\mathbf{I}_{a}^{b} c F(x) d x=c \mathbf{\coprod}_{a}^{b} F(x) d x .
$$

* Theorem I follows as a corollary of theorem VI for any function $F(x)$ known to be I-integrable from $a$ to $\dot{b}$.

$\dagger$ The content of a point-setlying on an infinite interval is defined in a footnote of $\$ 113^{\circ}$.

$\ddagger$ Even if $a b$ is an infinite interval. 
VII. If the functions $F(x), G(x)$ are $\mathbf{I}$-integrable from $a$ to $b$, so is the function $F(x)+G(x)$, and

$$
\mathbf{I}_{a}^{b}(F(x)+G(x)) d x=\mathbf{I}_{a}^{b} F(x) d x+\mathbf{I}_{a}^{b} G(x) d x .
$$

VIII. The transformation of definite integrals.

From the hypotheses:

1) The $x$-interval $a b$ and an $\bar{x}$-interval $\bar{a} \bar{b}$ (each interval being finite or infinite) are set in one-to-one point correspondence with preservation of sense by the transformation

where

$$
x=\phi(\bar{x})^{*} \quad(\bar{x} \text { on } \bar{a} \bar{b}),
$$

$$
a=\phi(\bar{a}), \quad b=\phi(\bar{b}) ;
$$

2) The $x$-set $\Xi_{a b}$ and an $\bar{x}$-set $\bar{\Xi}_{\bar{a} \bar{b}}$ correspond under the transformation (3);

3) On the $\bar{x}$-interval $\bar{a} \bar{b}$ apart from points $\bar{\xi}$ and limit-points $\bar{\xi}^{\prime}$ of $\bar{\Xi}_{\bar{a} \bar{b}}$ the function $\phi(\bar{x})$ has a continuous finite and non-zero derivative $\dagger \phi^{\prime}(\bar{x})$, follow the conclusions:

1) Under the transformation (3) the type $\mathbf{I}$ on the interval ab with respect to the set $\Xi_{a b}$ transforms into a type $\mathbf{I}$ on the interval $\bar{a} \bar{b}$ with respect to the set $\bar{\Xi}_{\bar{a} \bar{b}}$;

2) The function

$$
\bar{F}(\bar{x}) \equiv F(\phi(\bar{x})) \phi^{\prime}(x)
$$

is $\mathbf{I}$-integrable from $\bar{a}$ to $\bar{b}$;

3) The formula

$$
\mathbf{I}_{a}^{b} F(x) d x=\overline{\mathbf{I}}_{\bar{a}}^{\bar{b}} F(x) d x
$$

of transformation of definite integrals has validity.

$3^{\circ}$. These theorems, with the exception of the second, are valid for proper definite integrals in the usual sense; they belong to the elements of the theory.

* Where then $\phi(\bar{x})$ and its inverse $\bar{\phi}(x)$ are continuous functions of their respective arguments $\bar{x}, x$ on the respective intervals $\bar{a} \bar{b}, a b$. (Cf. the second footnote of $\S 11^{\circ}$.) It is to be noted that a point-set $\Omega$ of content zero transforms under a transformation (3) subject to the condition $1)$ into a point-set $\Omega$ likēwise of content zero.

$\dagger$ Which has then the sign of $(b-a) /(\bar{b}-\bar{a})$ as permanent sign. - No statement is made ccncerning the existence or properties of the derivative $\phi^{\prime}(\bar{x})$ at points $\bar{x}=\bar{\xi}, \bar{\xi}^{\prime}$.-If the function $\phi(\bar{x})$ satisfies the conditions 1$)-3$ ), the inverse function $\bar{\phi}(x)$ satisfies the corresponding conditions on the $x$-interval $a b$. Similarly there is a composition of transformations of this character. Here one needs to prove (indirectly) that $\bar{a}$ or $\bar{b}$ belongs to $\bar{\Xi}+\bar{\Xi}^{\prime}$ if it is infinite, so that, for example, no proper definite integral (over a finite interval) is (by theorem VIII) transformable into an integral over an infinite interval; this state of affairs accords with the general postulate of the close of $\S 1$. 
As to theorem VIII, cf. JoRDAN's Cours d' Analyse, ed. 2, vol. 1, nos. 141-144. The validity of all the theorems for proper integrals in the extended sense is easily perceived.

$4^{\circ}$. The I-integrals over infinite intervals ab are defined (cf. $\S 11^{\circ}$ ) as transforms of $\overline{\mathbf{I}}$-integrals over finite intervals $\bar{a} \bar{b}$ by the definite transformation $x=\psi(\bar{x})$ of $\S 113^{\circ}$.

That transformation (quâ $x=\phi(\bar{x})$ ) satisfies the conditions of theorem VIII on the $\bar{x}$-interval* $-1 \leqq \bar{x} \leqq+1$.

By the notion of composition of transformations (3) one readily proves that the general validity of the theorems of $\S 2$ follows from their validity for all finite intervals $a b, \bar{a} \bar{b}$ (the latter entering only in theorem VIII), and again that that validity follows from their validity for a single finite interval $a b$ with all finite intervals $\bar{a} \bar{b}$.

\section{$\S 3$.}

First definitional process of induction.

$1^{\circ}$. By the first process of induction we are to define (in $2^{\circ}-4^{\circ}$ ) from any set $\Xi_{0}$ and any type $\mathbf{I}$ of the set $\Xi$ a (related) type $\mathbf{I}_{\mathbf{z}_{0}}$ of the aggregate set $\Xi+\Xi_{0}$, that is, we are to characterize the functions $F(x) \mathbf{I}_{\mathbf{z}_{0}}$-integrable from $a$ to. $b$. We agree at once that if $a=b$ every function $F(x)$ is so integrable, the integral having the value zero.

We assume the validity of theorems I-VIII of $\S 2$ for the type $\mathbf{I}$, and (in $5^{\circ}-7^{\circ}$ ) we establish their validity for the type $\mathbf{I}_{\mathbf{E}_{0}}$.

In accordance with $\S 24^{\circ}$, in these definitions and proofs we may and do restrict attention to the case of finite intervals $a b$.

In $8^{\circ} \mathrm{I}$ indicate a necessary and sufficient condition for the existence of the $\mathbf{I}_{\Xi_{0}}$-integral, which is very useful in the construction of integrals of type $\mathbf{I}_{\Xi_{0}}$ from those of type $\mathbf{I}$.

$2^{\circ}$. The type $\mathbf{I}_{\Xi_{0}}$ depends upon the type $\mathbf{I}$ just as HaRNACK's general or narrow type $\dagger \int_{\left(\Xi_{0}\right)}$ depends upon the type $\int$ of proper definite integrals, except that now the set $\Xi_{0}$ is of arbitrary content $\gamma\left(\Xi_{0}\right)$ and does not necessarily lie on a finite interval. As far as convenient $I$ adopt the phrasings of my paper in the preceding number of this journal: On Haknack's Theory of Improper Definite Integrals, - and for brevity refer to it by the notation $H$. T.

$3^{\circ}$. The following notations are needed.

(a) With respect to any interval $a b$ and point-set $\Xi$ or interval-set $I$ the notation $\Xi_{a b}$ or $I_{a b}$ denotes the point-set of $\Xi$ or interval-set lying on $I$ which lies on $a b$.

* The extremities \pm 1 are the transforms of $\pm \infty$ and so belong to the closed set $\bar{\Xi}+\bar{\Xi}$.

† H. T. p. 304. 
$(\beta)$ When an interval-set * $I$ encloses a point-set $\Xi$ narrowly,* in which case it is denoted by the notation $I(\Xi)$, then its length $D_{I}$ is greater than the content $\gamma(\Xi)$ of $\Xi$; this positive difference $D_{I}-\gamma(\Xi)$ we call the $\Xi$-length of $I$, and denote it by $D(I, \Xi)$; on the set of all such interval-sets $I(\Xi)$ the lower limit of $D(I, \exists)$ is zero.

$(\gamma)$ With respect to an interval-set $I$ and a function $F(x)$ the function $F_{I}(x)$ is defined as having at every point $x$ of $I$ the value zero, and at every point $x$ not of $I$ the same determination as $F(x)$ at that point.

$(\delta)$ We denote the perfect set $\Xi_{0}+\Xi_{0}^{\prime}$ by $\Xi^{0}$.

$4^{\circ}$. Our function $F(x) \mathbf{I}_{z_{0}}$-integrable from $a$ to $b$ ( $a b$ unequal and finite) is, in the first place, I-integrable over every subinterval of $a \dot{b}$ containing no point $\xi^{0}$ of $\Xi^{0}$. This implies, according to theorems I, II, III, IV' for the type I, that $F_{I}(x)$ is $\mathbf{I}$-integrable from $a$ to $b$, where $I$ is any interval-set enclosing the point-set $\Xi_{a b}^{0}$ narrowly.

Then in the second place, the (finite) limit :

in notation $\dagger$

$$
\coprod_{D\left(I, \mathbb{Z}_{a b}^{0}\right)=0}^{I \mid I\left(\Xi^{0}\right)} \mathbf{I}_{a}^{b} F_{I}(x) d x,
$$

$$
\mathbf{I}_{a \Xi_{0}}^{b} F(x) d x
$$

exists ; that is, for every positive $\epsilon$ a positive $\delta_{\epsilon}^{1} \ddagger$ exists such that

$$
\left|\mathbf{I}_{a}^{b} F_{I}(x) d x-\mathbf{I}_{a \Xi_{0}}^{b} F(x) d x\right|<\epsilon
$$

for every interval-set $I$ enclosing $\Xi_{a b}^{0}$ narrowly and of $\Xi_{a b}^{0}$-length less than $\delta_{\epsilon}^{1}$.This limit is provably definite.-Thus in terms of the type $\mathbf{I}$ the type $\mathbf{I}_{\mathbf{\Xi}_{1}}$, has been completely defined.

It is to be noticed that

$$
\mathbf{I}_{x}^{b} F_{I}(x) d x=\mathbf{I}_{J_{a}^{b}} F(x) d x,
$$

where $J_{a}^{b}$ denotes the interval-set of $a b$ complementary to $I_{a b}$, its intervals taken in the sense $a b$, and where the notation on the right denotes the sum of the Iintegrals of $F(x)$ over these directed intervals of $J_{a}^{b}$, or zero if $J_{a}^{b}$ is non-existent. If in our definition we adopt $\mathbf{I}_{J_{a}^{b}} F(x) d x$ as the limitand expression, we have a form of definition closely analogous to that of JORDAN for the HaRNACKJORDAN integrals.

* H. T. $\S 15^{\circ}, 7^{\circ}$.

† The notation $\mathbf{I}_{\Xi_{0} a}^{b} F(x) d x$ would (except for its appearance) be preferable.

$\ddagger$ In this paper superscripts to $\delta_{\epsilon}$ notations are not exponents but mere affixes of discrimination. 
$5^{\circ}$. For the type $\mathbf{I}_{\Xi_{0}}$ (qua $\mathbf{I}$ ) the theorems I-VIII of $\S 2$ hold.-We admit the validity of these theorems for the type $I$, and, as.indicated in $1^{\circ}$, we suppose that $a$ and $b$ are finite, and furthermore distinct, since the theorems obviously hold if $a=b$. The truth of I and II for $\mathbf{I}_{\mathbf{z}_{0}}$ follows at once from their truth for $\mathbf{I}$. Theorems III, IV, IV', V, V', VI, VII for $\mathbf{I}_{\Xi_{0}}$ depend upon the lemmas given in $6^{\circ}$, essentially as the theorems H. T. $\S 3 \mathrm{~V}$, VIII, $\S 2$ III, $33 \mathrm{IX}, \mathrm{II}$, XVII depend upon the theorems H. T. $\S 3$ IV, V, VI. Theorem VIII is considered in $7^{\circ}$.

$6^{c}$. Lemma I. For every positive $\epsilon$ there is a positive $\delta_{\epsilon}^{2}$ such that

$$
\left|\mathbf{I}_{x_{1}}^{x_{2}} F_{I_{1}}^{\prime}(x) d x-\mathbf{I}_{x_{1}}^{x_{2}} F_{I_{2}}(x) d x\right|<\epsilon
$$

for every two points $x_{1} x_{2}$ of the finite interval $a b$ and every pair of interval-sets $I_{1} I_{2}$ each enclosing $\Xi_{a b}^{0}$ narrowly and of $\Xi_{a b}^{0}$-length less than $\delta_{\epsilon}^{2}$.- - Here the uniformity with respect to $x_{1} x_{2}$ is of central importance.

This lemma corresponds to $H$. T. $\S 3 \mathrm{IV}$, the proof of which was involved with that of a more general theorem $\mathrm{H}$. T. $\S 3 \mathrm{X}$, the general analogue of which is not now in question. I give as imple proof of the lemma.

The determination $\delta_{\epsilon}^{2}=\frac{1}{2} \delta_{\epsilon / 2}^{1}$ is effective.

If the lemma is not true, then for a certain determination of $\epsilon, x_{1}, x_{2}, I_{1}, I_{2}$ satisfying the specified conditions the inequality (1), call it $\left(1^{\prime}\right)$, is invalid. We may confine attention to the case $a<b, x_{1}<x_{2}$.

Let the interval-set $I$ enclose $\Xi_{a b}^{0}$ narrowly and have $\Xi_{a b}^{0}$-length less than $\delta_{\epsilon}^{2}$. Let the interval $a \beta(a<\beta)$ enclose $a b(a<b)$ so that

$$
a<a \leqq x_{1}<x_{2} \leqq b<\beta \text {. }
$$

Then each interval-set:

$$
I_{3}=I_{\alpha x_{1}}+I_{1 x_{1} x_{2}}+I_{x_{2} \beta}, I_{4}=I_{\alpha x_{1}}+I_{2 x_{1} x_{2}}+I_{x_{2} \beta},
$$

encloses $\Xi_{a b}^{0}$ narrowly and has* $\Xi_{a b}^{0}$-length less than $2 \delta_{\epsilon}^{2}=\delta_{\epsilon / 2}^{1}$.

Hence, obviously, recalling the definition, we have

$$
\left|\mathbf{I}^{b}{ }^{b} F_{I_{s}}(x) d x-\mathbf{I}_{a}^{b} F_{I_{4}}(x) d x\right|<\epsilon .
$$

On the other hand, by (2) and $\$ 2 \mathrm{IV}$, for the type $\mathbf{I}$, we have

$$
\mathbf{I}_{a}^{b} F_{I_{3}}(x) d x=\mathbf{I}_{a}^{x_{1}} F_{I}(x) d x+\mathbf{I}_{x_{1}}^{x_{2}} F_{I_{1}}(x) d x+\mathbf{I}_{x_{2}}^{b} F_{I}(x) d x
$$

* We observe that $I_{3}, I_{4}$ are parts of $I_{5}=I+I_{1}, I_{6}=I+I_{2}$ respectively and use the following theorem of the theory of contents :

If the interval-sets $I^{1}$ and $I^{2}$ enclose the point-sets $\Xi^{1}$ and $\Xi^{2}$ respertively, then the interval-set $I=I^{1}+I^{2}$ encloses the point-set $\Xi=\Xi^{1}+\Xi^{2}$, and

$$
D(I, \Xi) \leqq D\left(I^{1}, \Xi^{1}\right)+D\left(I^{2}, \Xi^{2}\right) \text {. }
$$


with a similar equation involving $I_{4}$ and $I_{2}$, so that

$$
\mathbf{I}_{a}^{b} F_{I_{3}}(x) d x-\mathbf{I}_{a}^{b} F_{I_{4}}(x) d x=\mathbf{I}_{x_{1}}^{x_{2}} F_{I_{1}}(x) d x-\mathbf{I}_{x_{1}}^{x_{2}} F_{I_{2}}(x) d x
$$

whence by $\left(1^{\prime}\right)$ we have, in contradiction with (3),

$$
\left|\mathbf{I}_{a}^{b} F_{I_{3}}(x) d x-\mathbf{I}_{a}^{b} F_{I_{4}}(x) d x\right| \geqq \epsilon .
$$

Thus lemma $I$ is proved.

Obviously lemma $I$ holds also if the interval-sets $I_{1} I_{2}$ enclose $\Xi_{._{1} x_{2}}^{0}$ (instead of $\left.\Xi_{a b}^{0}\right)$ narrowly, for the integrals in (1) are from $x_{1}$ to $x_{2}$. Hence by the usual limit-considerations theorem II is proved for the type $\mathbf{I}_{\mathbf{z}_{0}}$, and likewise

Lemma II. For every $\epsilon$ and every two points $x_{1} x_{2}$ of the finite interval $a b$

$$
\left|\mathbf{I}_{x_{1}}^{x_{2}} F_{I}(x) d x-\mathbf{I}_{x_{1} \Xi_{0}}^{x_{2}} F(x) d x\right| \leqq \epsilon
$$

for every interval-set $\mathbf{I}$ enclosing $\Xi_{a b}^{0}$ narrowly and of $\Xi_{a b}^{0}$-length less than $\delta_{\epsilon}^{2}$.

Lemma II is of fundamental importance in the deduction of theorems of linear form for the type $\mathbf{I}_{\Xi_{0}}$ from similar theorems for the type $\mathbf{I}$.

$7^{\circ}$. The truth of theorem VIII (with $a b, \bar{a} \bar{b}$ finite intervals) for the type $\mathbf{I}_{\Xi_{0}}$ (quâ $\mathbf{I}$ ) of the set $\Xi+\Xi_{0}$ (quâ $\Xi$ ) follows easily from its truth for the type I of the set $\Xi$.

We consider a transformation

$$
x=\phi(\bar{x})
$$

which satisfies the conditions of theorem VIII for the type $\mathbf{I}_{\Xi_{0}}$ of the set $\Xi+\Xi_{0}$.

Using notations of evident meaning we observe that the sets $\Xi_{0} \Xi^{0}$ correspond to similarly related sets $\Xi_{0} \bar{\Xi}^{0}$; that every $\bar{I}$ enclosing $\Xi^{0}$ narrowly corresponds to an $I$ enclosing $\Xi^{0}$ narrowly; that in the notation of $4^{\circ}$ every $\bar{J}_{\bar{a}}^{\bar{b}}$ corresponds to a $J_{a}^{b}$; and finally we observe, by considerations of the theory of contents of linear point-sets, in view of the continuity of the transformation (5) between $a b \bar{a} \bar{b}$, that for a positive $\epsilon$ a positive $\bar{\delta}_{\epsilon}^{1}$ exists such that the $\Xi_{a b}^{0}$-length of $I$ is less than $\delta_{\epsilon}^{1}$ in case the $\bar{\Xi}_{\bar{a} \bar{b}}^{0}$-length of $\bar{I}$ is less than $\delta_{\epsilon}^{1}$.

Then

$$
\left|\mathbf{I}_{J_{b}^{a}} F(x) d x-\mathbf{I}_{a \Xi_{0}}^{b} F(x) d x\right|<\epsilon
$$

for every interval-set $\bar{I}$ enclosing $\bar{\Xi}_{\bar{a} \bar{b}}^{0}$ narrowly and of $\bar{\Xi}_{a b}^{0}$ length less than $\bar{\delta}_{e}^{1}$; and simultaneously, for every (directed) interval $j$ of $J_{a}^{b}$,

$$
\coprod_{j} F(x) d x=\coprod_{j} \bar{F}(\bar{x}) d \bar{x},
$$

Trans. Am. Math. Soc. 31 
since the transformation (5) on $j \bar{j}$ satisfies the conditions of theorem VIII for the type $\mathbf{I}$ of the set $\Xi$; and thus

$$
\mathbf{I}_{J_{b}^{a}} F(x) d x=\overline{\mathbf{I}}_{\overline{\bar{a}} \bar{b}} \bar{F}(\bar{x}) d \bar{x} .
$$

From (6) and (8) by elimination we have the inequality needed to prove the validity of theorem VIII for the type $\mathbf{I}_{\Xi_{0}}$.

$8^{\circ}$. The theorem of $\mathrm{H}$. T. $\S 52^{\circ}$ may readily be generalized to the case of $\mathbf{I}_{\mathbf{E}_{0}}$-integrals. In the proof of the generalized theorem one uses instead of the theorem H. T. $\S 3 \mathrm{XIII}^{\prime}$ considerations of continuity and definitional considerations concerning the analogues of the integrals, loc. cit. (3). This theorem is of fundamental use in the construction (as in H. T. §5) from the functions $\psi_{01}(x)$, $\chi_{01}(x)$ of H. T. $\S 54^{\circ}$ of functions having integrals of type $I$ of a set $\Xi$ partitioned into a finite sequence $\Xi_{1}, \Xi_{2}, \ldots, \Xi_{m}$, viz.

$$
\mathbf{I} \equiv \int_{\Xi_{1} \Xi_{2} \ldots \Xi_{m}} .
$$

\section{$\S 4$.}

The second Definitional process of induction.

$1^{\circ}$. In the second process of induction we have to do with a simply ordered set* $M=\{m\}$ of symbols or marks $m$ and with a related simply ordered set $\left\{\mathbf{I}^{m}\right\}$ of types $\mathbf{I}^{m}$ of the point-set $\Xi^{m}$, where $\Xi^{m_{1}}$ is a proper subset of $\Xi^{m_{2}}$ if $m_{1}<m_{2}$.

We are to define in $4^{\circ}$ a type $\mathbf{I}^{M}$ of the aggregate set $\Xi^{M}$, where $\Xi^{M}$ denotes the (least) point-set containing every set $\Xi^{m}$ as a subset.

From this definition, on the assumption of the validity of theorems I-VIII of $\S 2$ for the types $\mathbf{I}^{m}$, follows their validity for the type $\mathbf{I}^{M}$, as one readily assures himself.

$2^{\circ}$. The simply ordered set $M=\{m\}$ may have a highest mark $m, m=m^{\prime}$; in this case we say that it terminates with the mark $m^{\prime}$. Every finite set $M$ terminates. A transfinite set $M$ may or may not terminate.

$3^{\circ}$. Even if the set $\left\{\Xi^{m}\right\}$ does not terminate the set $\left\{\Xi^{i n}\right\}$ may on an interval $i$ essentially terminate, i. e., there may be a mark $m_{0}$ such that $\Xi_{i}^{m} \equiv \Xi_{i}^{m_{0}}$ for every $m>m_{v}$, where $\Xi_{i}^{m}$ denotes the subset of $\Xi^{m}$ lying on $i$.

* With Cantor (Mathematische Annalen, vol. 46, p. 496) a set $M$ of marks $m$ is simply ordered if of every two distinct marks $m_{1} m_{2}$ one, say $m_{1}$, is distinguished, and if this distinction is transitive, i. e., if of the marks $m_{1} m_{2}$ the mark $m_{1}$ is distinguished, and of $m_{2} m_{3} m_{2}$ is distinguished, then of the marks $m_{1} m_{3}$ the mark $m_{1}$ is distinguished. One says that according to the ordering in question $m_{1}$ has the lower and $m_{2}$ the higher rank, in notation :

$$
m_{1} \prec m_{2}, \quad m_{2}>m_{1} .
$$


A point $x=x_{0}$ is with respect to the non-terminating transfinite set $\left\{\dot{E}^{m}\right\}$ critical, in case on no interval $i$ enclosing $x_{0}$ the set $\left\{\Xi^{m}\right\}$ is essentially a terminating set. We denote these critical points $x$ by the notation $\kappa$ and the set of all such points by the notation $K$. The set $K$ is closed.

If an interval $i$ contains no critical point, the set $\left\{\Xi^{m}\right\}$ on $i$ essentially terminates, as one proves by the usual interval-halving process.

Of course, the set $\mathrm{K}$ exists only if $\left\{\Xi^{m}\right\}$ is non-terminating; and even then it may contain no finite point.

$4^{\circ}$. We are to define an (existent) integral

$$
\coprod_{a}^{b M} F(x) d x
$$

of the type $\mathbf{I}^{M}$, that is, to characterize the function $F(x)$ in order that the symbol (1) may have a (definite finite) value.

If $b=a$, we give to the symbol (1) the value 0 .

If on the interval $a b$ the function $F(x)$ vanishes except perhaps at a set of points of content zero, we give to the symbol (1) the value 0 .

Further, if the interval $a b$ contains no critical point $\kappa$ and so on $a b$ the set $\left\{\Xi^{m}\right\}$ is essentially terminating, and if there is a mark $m_{0}$ such that for all marks $m, m \geqq m_{0}$, the $\mathbf{I}^{m}$-integrals of $F(x)$ from $a$ to $b$ exist, and for every subinterval $a^{\prime} b^{\prime}$ of $a b$ and mark $m>m_{0}$

$$
\mathbf{I}_{a^{\prime}}^{b^{\prime} m} F(x) d x=\mathbf{I}_{a^{\prime}}^{b^{\prime} m_{0}} F(x) d x,
$$

then we denote by $\mathbf{I}_{a}^{b M} F^{\prime}(x) d x$ the common value of the existent integrals

$$
\mathbf{I}_{a}^{b m} F(x) d x
$$$$
\left(m \geqq m_{0}\right) \text {. }
$$

Finally, if the finite or infinite interval $a b$ splits into a finite number $n$ of intervals $a_{k} b_{k}(k=1, \ldots, n)$ taken in the sense of $a b$ in such a way that by the specifications already made the integrals $\mathbf{I}_{a_{k}}^{b_{k} M} F(x) d x$ are defined, we set

$$
\mathbf{I}_{a}^{b M} F(x) d x \equiv \sum_{k=1}^{n} \mathbf{I}_{a_{k}}^{b_{k} M} F(x) d x
$$

Thus the symbol (1) is given, if any, in fact a definite finite value.

It is clear that if the set $M$ terminates, with $m$ say, then the types $\mathbf{I}^{M} \mathbf{I}^{m}$ are identical.

$$
\S 5 \text {. }
$$

The Definition of the general tyPe I OF THE SET $\Xi$.

$1^{\circ}$. With Cantor* a well ordered set $W=\{w\}$ of marks $w$ is a simply ordered set with the property that its every subset (including the set itself) has a mark

\footnotetext{
* Mathematische Annalen, vol. 49 (1897), pp. 207, 208.
} 
of lowest rank. Every subset of $W$ is itself a well ordered set. I denote by $W_{1}=\left\{w_{1}\right\}$ the set of all marks $w=w_{1}$ having immediately preceding marks $w=w_{1}^{0}$; the mark of lowest rank by $w_{0}$; and the residual set by $W_{2}=\left\{w_{2}\right\}$. It is to be noticed that the marks $w=w_{1}^{0}$ include all the marks of $W$ except the terminal mark, if there is one.

$2^{\circ}$. For the definition of the general type $I$ of improper integrals with respect to the point-set $\Xi I$ consider the general well ordered set $W$ such that $\Xi$ admits an exhibition, in the notation of $\S 41^{\circ}$, of the form $\Xi \equiv \Xi^{W}$, and $I$ consider the general exhibition of $\exists$ in this form. The understanding is, however, that $\Xi^{w_{0}} \equiv\{ \pm \infty\}$ and that for every $w_{2}$ the set $\Xi^{w_{2}}$ is the least set containing as subsets the sets $\Xi^{w}, w<w_{2}$.

We set $\Xi_{v_{1}} \equiv \Xi^{x_{1}}-\Xi^{1_{1}^{0}}$; thus $\Xi_{w_{1}}$ denotes an existing point-set.

$3^{\circ}$. From the type $I^{w_{1}^{0}}$ of the set $\Xi^{w_{1}^{0}}$ with the properties I-VIII of $\S 2$ and the set $\Xi_{x_{1}}$ we can, by the first process of induction, define the type $\mathbf{I}^{w_{1}}$ of the set $\Xi^{w_{1}}$ with the same properties.

Likewise from the types $\mathbf{I}^{10}, w<w_{2}$, of the respective sets $\Xi^{w}$ we can define the type $\mathbf{I}^{w_{2}}$ of the set $\Xi^{w_{2}}$ by the second process of induction.

Likewise from all the types $\mathbf{I}^{w}, w$ of $W$, of the respective sets $\Xi^{w}$ we can define the type $\mathbf{I}^{W}$ of the set $\Xi{ }^{W} \equiv \Xi$ by the second process.

$4^{\circ}$. Now we take as type $I^{w_{0}}$ of the set $\Xi^{\dot{w}_{0}} \equiv\{ \pm \infty\}$ on finite intervals the (extended) type of proper definite integrals defined in $\S 116^{\circ}$. From this type follow the definitions of all the types $\mathbf{I}^{w}$ and of the type $\mathbf{I}^{w}$. For, if not, there is an undefined type $\mathbf{I}^{w^{\prime}}$ of lowest rank $w^{\prime}$; and this is impossible, for, by the statements just made, $w^{\prime}$ is neither $w_{0}$ nor a $w_{1}$ nor a $w_{2}$. - Thus the general type I of the set $\Xi$ has definition uniquely related to the general exhibition of $\Xi$ in the specified partitional form $\Xi \equiv \Xi^{W}$, where $W$ denotes a well ordered set and where similar well ordered sets $W$ are for the purposes of the definition equivalent.

$5^{\circ}$. In conclusion, we point out how the system of types of improper integrals thus defined embraces the four current types as extended $\left(\S 11^{\circ}, 16^{\circ}\right)$.

Type I.-In the first type the set $\Xi$ is reducible, that is, for some (lowest) ordinal $a \geqq 1$ of CANTOR's first or second class of ordinals the derived set $\Xi^{(a)} \equiv 0$. We consider the set $\{\beta\}$ of ordinals $\beta, 1 \leqq \beta<a$, arranged in their natural ascending order. This set $\{\beta\}$ is a well ordered set. We consider the similar well ordered set $\left\{w_{1 \beta}\right\}$ and make it the set $W_{1}$ of a well ordered set $W, W=w_{0}+W_{1}+W_{2}$, by introducing the initial mark $w_{0}$ and a set $W_{2}$ of marks $w_{2}=w_{2 \beta_{2}}$; here $\beta_{2}$ denotes an ordinal $>1$ having no immediately preceding ordinal, while in well ordered set $W$ the mark $w_{2 \beta_{2}}$ immediately precedes the mark $w_{1 \beta_{2}}$. Then the set $\Xi^{w_{0}}$ is $\{ \pm \infty\}$, the set $\Xi^{w_{1 \beta}}$ contains all 
points $\xi$ not belonging to the derived set $\Xi^{(\beta)}$, and the set $\Xi^{w_{2} \beta_{2}}$ is the least set containing as subsets the sets $\Xi^{w_{1 \beta}}, \beta<\beta_{2}$, and so the least set containing as subsets the sets $\Xi^{w 0}, w<w_{2_{\beta_{2}}}$. The set $\Xi^{\prime w^{\prime}}$ is a proper subset of $\Xi^{\prime w^{\prime \prime}}$ if $w^{\prime}<w^{\prime \prime}$.

TrPe II.-In the second type the set $\exists$ is any set containing $\pm \infty$; the set $W$ consists of two ordered elements $w_{0}, w_{1}$; and $\Xi^{w_{0}}=\{ \pm \infty\}, \Xi^{w_{1}}=\Xi$.

TyPE III.-The third type is essentially the same as the first type.

TrPe IV.-The fourth type is in a sense (sufficiently indicated in $\S 19^{\circ}$ ) a combination of the second and the first types.

The University of Chicago,

October 2, 1901. 